\title{
Neuro-mechanical and metabolic adjustments to the repeated anaerobic sprint test in professional football players
}

\author{
Franck Brocherie · Gregoire P. Millet • Olivier Girard
}

Received: 24 June 2014 / Accepted: 27 November 2014 / Published online: 7 December 2014

(C) Springer-Verlag Berlin Heidelberg 2014

\begin{abstract}
Purpose This study aimed to determine the neuromechanical and metabolic adjustments in the lower limbs induced by the running anaerobic sprint test (the so-called RAST).

Methods Eight professional football players performed $6 \times 35 \mathrm{~m}$ sprints interspersed with $10 \mathrm{~s}$ of active recovery on artificial turf with their football shoes. Sprinting mechanics (plantar pressure insoles), root mean square activity of the vastus lateralis (VL), rectus femoris (RF), and biceps femoris (BF) muscles (surface electromyography, EMG) and VL muscle oxygenation (near-infrared spectroscopy) were monitored continuously.

Results Sprint time, contact time and total stride duration increased from the first to the last repetition $(+17.4,+20.0$ and $+16.6 \%$; all $P<0.05$ ), while flight time and stride length remained constant. Stride frequency $(-13.9 \%$; $P<0.001)$ and vertical stiffness decreased $(-27.2 \%$; $P<0.001)$ across trials. Root mean square EMG activities of RF and BF $(-18.7$ and $-18.1 \% ; P<0.01$ and 0.001 , respectively), but not VL $(-1.2 \% ; P>0.05)$, decreased over sprint repetitions and were correlated with the increase in running time $(r=-0.82$ and -0.90 ; both $P<0.05)$. Together with a better maintenance of RF and BF muscles
\end{abstract}

Communicated by Peter Krustrup.

F. Brocherie $(\varangle) \cdot$ G. P. Millet · O. Girard

ISSUL, Department of Physiology, Faculty of Biology

and Medicine, University of Lausanne, Building Geopolis,

Campus Dorigny, 1015 Lausanne, Switzerland

e-mail: franck.brocherie@unil.ch

O. Girard

Athlete Health and Performance Research Centre, ASPETAR -

Qatar Orthopaedic and Sports Medicine Hospital, Doha, Qatar activation levels over sprint repetitions, players with a better repeated-sprint performance (lower cumulated times) also displayed faster muscle de- (during sprints) and reoxygenation (during recovery) rates $(r=-0.74$ and -0.84 ; $P<0.05$ and 0.01 , respectively).

Conclusion The repeated anaerobic sprint test leads to substantial alterations in stride mechanics and leg-spring behaviour. Our results also strengthen the link between repeated-sprint ability and the change in neuromuscular activation as well as in muscle de- and re-oxygenation rates.

Keywords Repeated-sprint ability · Spring-mass model characteristics $\cdot$ Electromyographic activity $\cdot$ Near-infrared spectroscopy $\cdot$ Football

$\begin{array}{ll}\text { Abbreviations } \\ \text { BF } & \text { Biceps femoris } \\ \text { EMG } & \text { Electromyography } \\ F z_{\text {max }} & \text { Maximal vertical force } \\ K_{\text {leg }} & \text { Leg stiffness } \\ K_{\text {vert }} & \text { Vertical stiffness } \\ L_{0} & \text { Lower-limb length } \\ \text { RAST } & \text { Running anaerobic Sprint Test } \\ \text { RF } & \text { Rectus femoris } \\ \text { RSA } & \text { Repeated-sprint ability } \\ \text { RMS } & \text { Root mean square } \\ S_{\text {dec }} & \text { Sprint decrement score } \\ S_{\mathrm{F}} & \text { Stride frequency } \\ S_{\mathrm{L}} & \text { Stride length } \\ t_{\mathrm{c}} & \text { Contact time } \\ t_{\mathrm{f}} & \text { Flight time } \\ \text { TSI } & \text { Tissue saturation index } \\ V_{\text {forward }} & \text { Forward running velocity } \\ \text { VL } & \text { Vastus lateralis } \\ & \end{array}$


$\Delta L \quad$ Leg compression

$\Delta z \quad$ Centre of mass vertical displacement

\section{Introduction}

During competition, a football player's physical performance is typically characterized by total distance covered in a game, total sprint distance and execution of technical skills (e.g. pass, shot or cross success). While reductions in total distance will alter match play characteristics, gamedefining events are often correlated to successful integration of sprinting and technical capacities. For example, a straight sprint is most often seen prior to a goal being scored (Faude et al. 2012). Unsurprisingly, research pertaining to the factors compromising fatigue resistance during tests of repeated-sprint ability (RSA), closely resembling team-sport activity patterns, has grown enormously in the past few years. Laboratory cycling (Mendez-Villanueva et al. 2008; Racinais et al. 2007) and, to a lesser extent, running on a treadmill (Buchheit et al. 2009; Delextrat et al. 2013; Morin et al. 2011) have traditionally been employed as RSA test modalities. While the use of cycle ergometry as the mode of exercise has limited applications to team sports, laboratory-based RSA protocols allow a deeper understanding of the neuro-mechanical and metabolic manifestation of fatigue. For instance, measuring directly ground reaction forces, Morin et al. (2011) reported significant decrease in the capability to produce total force and larger decrease in the ability to apply it in a horizontal orientation during acceleration when maximal 6-s sprints with incomplete recoveries were repeated. However, instantaneous speed curves obtained on a motorized treadmill with constant motor torque (recently validated for sprint use) were on average $20 \%$ slower than over-ground (standard track) during 100-m sprint (Morin and Seve 2011)

Owing to the 'sport-specific' principle, many RSA evaluations of football players have been field based. Generally, these investigations have hitherto-studied players' RSA from a split-time point of view with performance (i.e. initial sprint, cumulated sprint times) or fatigue resistance (i.e. sprint decrement score, fatigue index) often being the only available outcome variables. Nonetheless, pressure insoles have also been used successfully to estimate how running mechanics (i.e. running kinematics and spring-mass model) are regulated in a group of thirteen U19 football players completing six $20-\mathrm{m}$ sprints with $20 \mathrm{~s}$ of passive recovery (Girard et al. 2011c). Using this approach, we reported that repeated sprinting on natural grass with players wearing their football boots induces specific impairments in sprinting mechanics (e.g. monotonic decreases in vertical stiffness stemming from progressively slower stride frequency) as neuromuscular function in the lower limbs is weakened (Girard et al. 2011c); this may not occur (or to a different extent) when players are tested in other conditions. Hence, RSA variables are affected by floor surface and footwear, with higher performance achieved with players wearing sneakers on a track compared to football cleats on grass (Kalva-Filho et al. 2013). It is therefore imperative to test players in their competition environment in order to maximize ecological validity of the research findings and derive scientifically sounded recommendations.

While neuromuscular factors (i.e. neural drive and motor units recruitment strategies determining peak sprinting speed) are key determinants of one individual's RSA, metabolic factors related with the control of highenergy phosphate (adenosine triphosphate and phosphocreatine) also play a decisive role in delaying fatigue development during RSA test (Girard et al. 2011a). With the development of non-invasive and rather inexpensive portable near-infrared spectroscopy (NIRS) devices, it is now possible to routinely assess tissue oxygenation status of locomotor muscles (Ferrari et al. 2011). Besides muscle de-oxygenation during exercise, faster pulmonary oxygen uptake (Dupont et al. 2010a) and muscle re-oxygenation (Buchheit et al. 2009) during recovery periods between sprints have also been associated with improved performance during successive sprints. Furthermore, the implementation of an 8-wk endurance-oriented training programme, which improved maximal aerobic speed and endurance capacity, accelerated post-sprints muscle reoxygenation rates (Buchheit and Ufland 2011). Considering that post-sprint muscle re-oxygenation rate and phosphocreatine stores replenishment (i.e. a major determinant of RSA) share similar recovery kinetics (i.e. at least during submaximal exercise; McCully et al. 1994), the importance of muscle re-oxygenation rates for improving RSA is paramount.

In the team-sport literature, there is a large diversity of run-based RSA protocols with differences in terms of sprint duration (4-6 s) or distance (10-40 m), sprint number (515 repetitions), recovery time (10-30 s) or nature (passive or active) (Glaister et al. 2008; Spencer et al. 2005). While participant characteristics or sport-specific considerations complicate the emergence of 'gold standards' for the selection of RSA test, the popularity of the so-called Running Anaerobic Sprint Test or RAST (i.e. $6 \times 35 \mathrm{~m}$ with $10 \mathrm{~s}$ rest) (Zagatto et al. 2009) has grown in recent years in both the scientific (Brocherie et al. 2014b; Cipryan and Gajda 2011; Deminice et al. 2013; Keir et al. 2013) and coaching (particularly in South America) communities. Considering that this RSA field-based test is reliable and valid for team sports (Balciunas et al. 2006), potentially a good performance predictor of anaerobic power (Zagatto et al. 2009) and short-duration runs (35-400 m) (Beck et al. 2014), its 
implementation in football training and/or testing routines might be relevant.

The purpose of the present study was therefore to determine changes in sprinting mechanics, lower-limb neuromuscular activity and vastus lateralis (VL) muscle de-/ re-oxygenation rates in a cohort of professional football players wearing their football cleats on grass while performing the so-called RAST ( $35 \mathrm{~m}$ efforts repeated six times with short between-sprint recovery periods). With an exercise-to-rest ratio of 1:2, we hypothesized that the inability to maintain sprint performance during the RAST will be substantial (sprint decrement score $>8 \%$ ), which would be evidenced biomechanically by less efficient strides throughout sprints. We further hypothesized that those players presenting neuromuscular alterations (i.e. larger fatigue-induced reductions in net motor unit activity) of the lower limb and/or slower muscle re-oxygenation rates would also display a poorer ability to maintain sprint performance constant across repetitions.

\section{Methods}

\section{Subjects}

Eight international male football outfield players (mean $\pm \mathrm{SD}$; age $27.8 \pm 5.0$ years, body weight $71.6 \pm 3.3 \mathrm{~kg}$, stature $176.1 \pm 4.1 \mathrm{~cm}$ ) belonging to the national 'A' squad of the Qatar Football Association were involved in this study. All players had a minimum of 5 years of experience in the domestic 1st division league (i.e. Qatar Star League), with an average participation of $10-12 \mathrm{~h}$ of training and competitive play per week (5-6 football training sessions, 1 strength training session, 1-2 conditioning sessions, 1-2 domestic games per week). The procedure complied with the declaration of Helsinki regarding human experimentation and was approved by the local scientific and Ethics Committee. All players were provided with the procedures, including risks associated with participation in the study, and written informed consent was obtained. According to the medical examination performed before the beginning of testing, the participants were not presenting recent muscular or joint injuries, receiving any medication or wearing orthotics that could interfere with their running pattern.

\section{Study overview}

All participants were tested at the end of the competitive season (May-June 2012) between 5 and $7 \mathrm{pm}$ at $22.0 \pm 0.5{ }^{\circ} \mathrm{C}$ and $55 \pm 2 \%$ relative humidity. Initially, the total body mass of the subjects was measured, including the garments worn during the tests on a digital scale
(Seca 769, Hamburg, Germany). After a standardized warm-up of $\sim 20$ min including 5 min of low-intensity running, 10 min of athletic drills (e.g. skipping, high knee runs, 3 shorts bursts of progressive accelerations on the track and $2 \times$ maximal 10-m straight-line sprints) followed by 2 min of rest, the participants performed the RAST test; e.g. $6 \times 35 \mathrm{~m}$ maximal sprints with a $10 \mathrm{~s}$ interval between each sprint. Participants were instructed to sprint as fast as possible over the $35-\mathrm{m}$ distance, while strong verbal encouragements were provided by experimenters and team members to ensure the maintenance of maximum effort. Players were familiar with the RAST testing procedures, which is an integral part of their evaluation procedure. The test was conducted on an indoor football field (artificial turf; Classic series, G3, Mondo, Italy), while players were wearing their own football boots. Stride mechanics (springmass model; SMM), lower-limb muscle activation (surface electromyography) and VL muscle oxygenation (NIRS) were evaluated.

\section{Running anaerobic sprint test}

The RAST protocol was followed according to the guidelines developed at the University of Wolverhampton (UK), and as reported previously (Zagatto et al. 2009). Briefly, the RAST involved $6 \times 35 \mathrm{~m}$ straight-line maximal sprints in alternating directions interspersed by $10 \mathrm{~s}$ of recovery. After deceleration until a cone placed at $10 \mathrm{~m}$, players jogged back to the starting line and assumed a standing ready position for $2 \mathrm{~s}$ before the next sprint. A countdown was announced before each sprint (i.e. ' 5 s, 3-2-1, Go').

Sprinting times were measured to the nearest $0.01 \mathrm{~s}$ using dual-beam electronic timing gates (TAC System, TT Sport, Galazzano, Republic of San Marino), which height was adjusted according to the height of the participant's hip and placed at $0,17.5$ and $35 \mathrm{~m}$. Each sprint was initiated from an individually chosen standing position with their leg foot in front, $50 \mathrm{~cm}$ behind the first timing gate. RSA was assessed using three scores: the fastest (i.e. initial in all cases) sprint time, the cumulated sprint time (i.e. sum of the six sprints) and the sprint decrement score $\left[S_{\mathrm{dec}}(\%)=\right.$ $100-[($ fastests print time $\times 6) /($ cumulated sprint $\times$ times $)]$ $\times 100]$ (Glaister et al. 2008).

The power in each run was determined through time, distance and body mass $[$ Power $(\mathrm{W})=$ (body mass $\times$ distance $^{2} /$ Time $^{3}$ ] (Kalva-Filho et al. 2013). The peak power, mean power and minimum power, presented both in absolute and normalized (i.e. relative to body mass) values, as well as the fatigue index fatigue index $(\%)=($ peak power - minimum power $) \times 100$ /peak power] were adopted as the RAST variables (KalvaFilho et al. 2013). High reliability $(r=0.90)$ has been reported in young basketball players (Balciunas et al. 2006) 
or in armed force members (intra- class correlations $>0.65$ ) (Zagatto et al. 2009) using the RAST.

Lactate and perceptual response

Immediately after the RSA test, subjects were seated on a chair for 4 min before a fingertip blood sample was collected for blood lactate concentration determination (Accutrend Plus System, Roche Diagnostics, Mannheim, Germany). The accuracy of the analyzer was checked before each test using standards. Rating of perceived exertion was assessed with the 6-20 Borg scale (Borg 1970) exactly $30 \mathrm{~s}$ after exercise termination.

Data acquisition and analysis

Each sprint trial included 19-21 ground contacts. After excluding the two last ground contacts, the remaining last three consecutive steps, i.e. representative of the 25-30-m distance interval, were used for final analysis (i.e. running mechanics and EMG data).

\section{Running mechanics}

Insole plantar loading of both feet was recorded using the X-Pedar Mobile insole (Novel GmbH, Munich, Germany) consisting of a 2-mm thick array of 99 capacitive pressure sensors, as previously described (Girard et al. 2011c). Briefly, flight $\left(t_{\mathrm{f}}\right)$ and contact $\left(t_{\mathrm{c}}\right)$ times were determined at a sampling frequency of $100 \mathrm{~Hz}$. Stride frequency $\left[S_{\mathrm{F}}(\mathrm{Hz})=1 /\left(t_{\mathrm{c}}+t_{\mathrm{f}}\right)\right]$ and stride length $\left[S_{\mathrm{L}}(\mathrm{m})=V_{\text {forward }} / S_{\mathrm{F}}\right]$ were calculated.

From these measurements of $t_{\mathrm{f}}$ and $t_{\mathrm{c}}$, forward running velocity ( $V_{\text {forward }}$ at the $17.5-35-\mathrm{m}$ distance interval in $\mathrm{m} \mathrm{s}^{-1}$ ) and from participants' body mass ( $\mathrm{m}$ in $\mathrm{kg}$ ) and lower-limb length ( $L_{0}$ in $\left.\mathrm{m}\right)$, SMM parameters were determined using the computation method proposed by Morin et al. (Morin et al. 2005). This method, based on a modelling of the ground reaction force signal, during the contact phase by a sine function (Morin et al. 2005) was recently shown to provide reasonable estimates when compared with direct kinetic-kinematic analysis (Coleman et al. 2012). Vertical stiffness $\left(K_{\text {vert }}\right.$ in $\left.\mathrm{kN} \mathrm{m}^{-1}\right)$ was calculated as the ratio of the maximal vertical force $\left(F z_{\max }\right.$ in $\left.\mathrm{N}\right)$ to the maximal downward displacement of the centre of mass (CM) during contact ( $\Delta z$ in $\mathrm{m})$ :

$K_{\text {vert }}=F z_{\max } / \Delta z$

with

$\left.F z_{\max }=\operatorname{mg}(\pi / 2)\left[\left(t_{\mathrm{f}} / t_{\mathrm{c}}\right)+1\right)\right]$

and

$\Delta z=-\left(\mathrm{F} z_{\max } / m\right)\left(t_{\mathrm{c}}^{2} / \pi^{2}\right)+\mathrm{g}\left(t_{\mathrm{c}}^{2} / 8\right)$
Leg stiffness $\left(K_{\text {leg }}\right.$ in $\left.\mathrm{kN} \mathrm{m}^{-1}\right)$ was calculated as the ratio of $F z_{\max }$ to the peak displacement of the leg spring (leg compression) during contact ( $\Delta L$ in $\mathrm{m})$ :

$K_{\text {leg }}=F z_{\text {max }} / \Delta L$

with

$\Delta L=L_{0}-\sqrt{\left[L_{0}^{2}-\left(V_{\text {forward }} t_{\mathrm{c}} / 2\right)^{2}\right]}+\Delta z$

It was assumed that the vertical velocity of $\mathrm{CM}$ was zero at the time of $F z_{\max }$. In this equation, $L_{0}$ (initial leg length from great trochanter to ground distance in a standing position) was determined from participant's stature as $L_{0}=0.53 \times$ stature (Winter 1990).

Near-infrared spectroscopy

The portable NIRS apparatus (Portamon, Artinis, Medical System, Zetten, The Netherlands) used in this study was a 2 -wavelength continuous wave system, which procedure used to collect data was the same as described previously with a similar portable device (Buchheit et al. 2009, 2010). Briefly, NIRS probes were positioned on the right VL muscle belly (approximately $10 \mathrm{~cm}$ above the proximal border of the patella) using a black, plastic spacer with optode distance of $4.5 \mathrm{~cm}$. The probe was held in place via doublesided, stick discs and black bandages to reduce the intrusion of extraneous light and the loss of transmitted NIRS light from the field of investigation.

A modified form of the Beer-Lambert law was used to determine micromolar changes in tissue oxyhemoglobin, deoxyhemoglobin and total hemoglobin using the differences in absorption characteristics of light at 750 and $850 \mathrm{~nm}$. Given the uncertainty of the proton pathlength at rest and during exercise, we used an arbitrary value for the differential pathlength of 3.83 (Buchheit et al. 2010). Muscle oxygenation in terms of tissue saturation index (TSI, expressed in \% and calculated as oxyhemoglobin/(oxyhemoglobin + deoxyhemoglobin) $\times 100$ ) was monitored (Ihsan et al. 2013). The NIRS system was connected to a personal computer by Bluetooth for data acquisition $(10 \mathrm{~Hz})$, analogue-to-digital conversion and subsequent analysis.

Data were averaged on 1-s basis, and a moving average $(3 \mathrm{~s})$ was applied to smooth the signal. In our study, we decided to restrict our NIRS analyses to TSI because it provides a better indication of muscle oxygenation status when blood flow is not constant (i.e. during exercise, principally due to a muscle pump effect) (Wolf et al. 2007). TSI reflects the dynamic balance between $\mathrm{O}_{2}$ supply and $\mathrm{O}_{2}$ consumption and is independent of near-infrared photon pathlength in muscle tissue.

Prior to the commencement of the RAST, subjects stood still for a 30-s period during which baseline TSI was 
Fig. 1 Changes in tissue saturation index (vastus lateralis, TSI; top panel) and corresponding muscle de-oxygenation (sprint) and re-oxygenation (recovery) for each of the six $35 \mathrm{~m}$ sprint repetition during the RAST (bottom panel). De-oxygenation and re-oxygenation rates are the change in TSI from the minimum and maximum values during work/rest intervals.

* Significantly different from sprint $1, P<0.05 ; * * P<0.01$

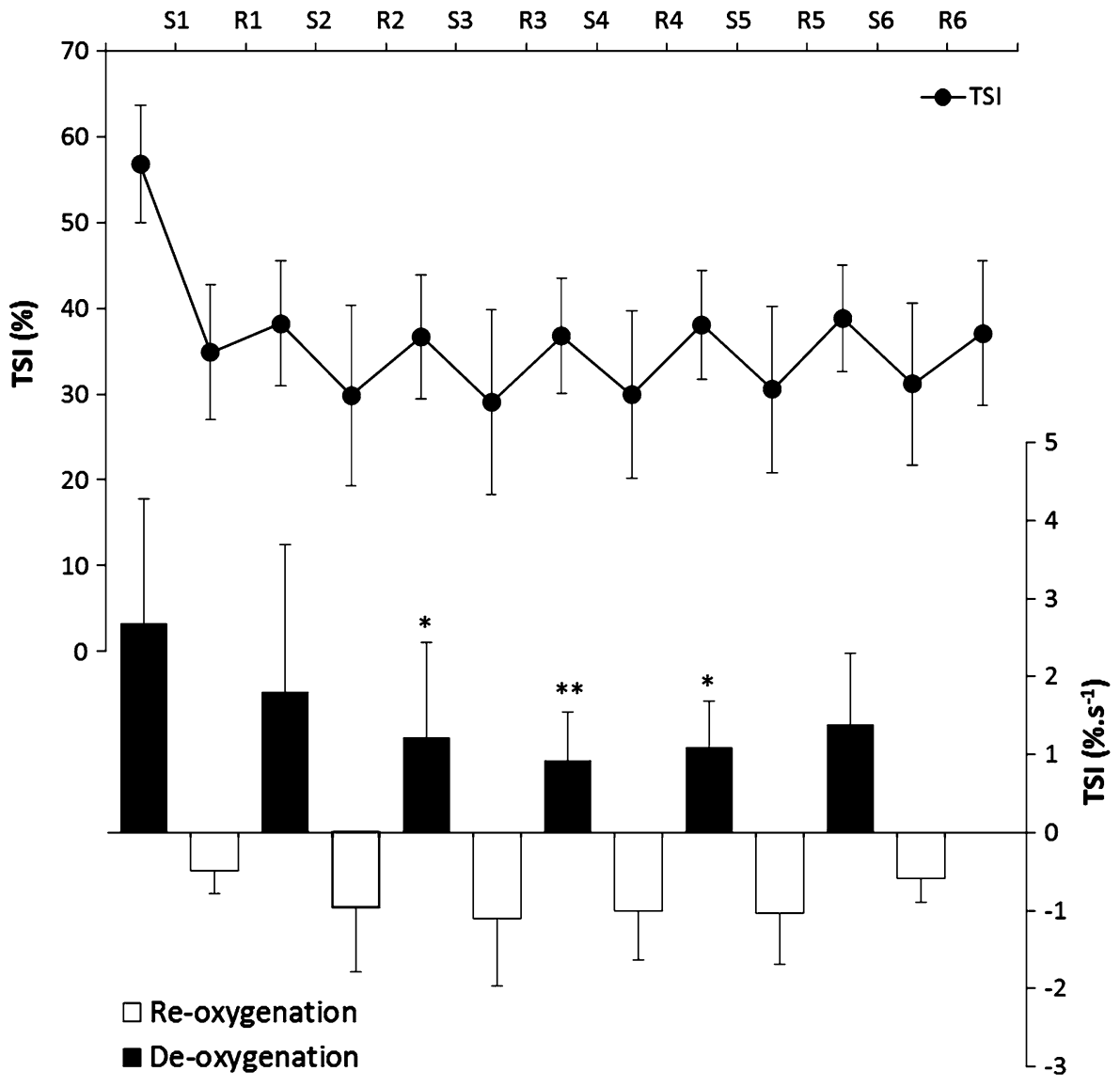

established. During the RAST, absolute maximal and minimal TSI values within every work and recovery intervals were first extracted (Fig. 1). We further calculated the VL de-oxygenation rate as the difference between the maximum (work interval) and minimum (rest interval) TSI values, divided by the work interval duration (i.e. the exact sprinting time; $\sim 5 \mathrm{~s}$ ). Similarly, re-oxygenation rate was determined as the difference between the minimum (rest interval) and the maximum (work interval) TSI values, divided by the rest interval duration (i.e. $\sim 10 \mathrm{~s}$ ). A recent comparison of three methods (linear modelling, minimum and maximum values during work/rest intervals and mean values during work/rest intervals) to analyse muscle de- and re-oxygenation rates has shown that, during intermittent exercise, linear modelling and minimum-maximum values (coefficient of variation $=7.2$ and $9.3 \%$, respectively) were the most reliable analysis methods (Ihsan et al. 2013). To further characterise the TSI response during the RAST, we also averaged de- and re-oxygenation rates values across the six sprints.

\section{EMG activity}

Electromyographic (EMG) signals from superficial VL, rectus femoris (RF) and biceps femoris (BF) muscles of the right lower limb were recorded using pre-amplified bi-polar surface EMG (Delsys, Trigno Wireless, Boston, Massachusetts, USA) with an inter-electrode (centre-to-centre) distance of $20 \mathrm{~mm}$ and placed according to the surface electromyography for the non-invasive assessment of muscles (SENIAM) project's recommendations. Before electrode placement, the skin was lightly abraded and washed to remove surface layers of dead skin, hair, and oil. All electrodes were secured with elastic cohesive bandage to reduce any movement of electrodes during the run or artefact in the signal. The EMG signal was amplified and filtered (bandwidth $20-450 \mathrm{~Hz}$, gain $=1,000$, sampling frequency $2,000 \mathrm{~Hz}$ ) and recorded using Delsys hardware (Delsys Inc, Boston, MA, USA). For each individual, a burst of muscle activity was identified from the same strides as SMM parameters (i.e. during the three strides preceding the two last ground contacts) as the amplitude of muscle activity exceeding $15 \%$ of peak activation for more than $10 \%$ of the stride (Kelly et al. 2010). For each sprint, the root mean square (RMS) activity was normalized to the first sprint value, which was assigned the value of $100 \%$ (Mendez-Villanueva et al. 2008).

\section{Statistical analysis}

Data are presented as mean \pm SD. Normal distribution of the data was tested using the Shapiro-Wilk test. A one-way 
Table 1 Running anaerobic sprint test (RAST) results

\begin{tabular}{|c|c|c|c|c|c|c|c|c|}
\hline \multicolumn{2}{|l|}{$P_{\text {peak }}$} & \multirow{2}{*}{$\begin{array}{l}P_{\text {mean }} \\
(\mathrm{W})\end{array}$} & \multirow{2}{*}{$\begin{array}{l}P_{\text {mean }} \\
\left(\mathrm{W} \mathrm{kg}^{-1}\right)\end{array}$} & \multirow{2}{*}{$\begin{array}{l}P_{\min } \\
(\mathrm{W})\end{array}$} & \multirow{2}{*}{$\begin{array}{l}P_{\min } \\
\left(\mathrm{W} \mathrm{kg}^{-1}\right)\end{array}$} & \multirow{2}{*}{$\begin{array}{l}\text { FI } \\
(\%)\end{array}$} & \multirow{2}{*}{$\begin{array}{l}{[\mathrm{La}]} \\
\left(\mathrm{mmol} \mathrm{L}^{-1}\right)\end{array}$} & \multirow{2}{*}{$\begin{array}{l}\text { RPE } \\
\text { (a.u.) }\end{array}$} \\
\hline$(\mathrm{W})$ & $\left(\mathrm{W} \mathrm{kg}^{-1}\right)$ & & & & & & & \\
\hline $759.4 \pm 64.3$ & $10.6 \pm 0.9$ & $592.9 \pm 69.9$ & $8.3 \pm 0.9$ & $467.6 \pm 74.4$ & $6.5 \pm 1.0$ & $38.6 \pm 7.2$ & $10.5 \pm 2.0$ & $15.9 \pm 0.9$ \\
\hline
\end{tabular}

Values are mean $\pm \mathrm{SD}$

Peak power $\left(P_{\text {peak }}\right)$, mean power $\left(P_{\text {mean }}\right)$, minimum power $\left(P_{\text {min }}\right)$, fatigue index $(\mathrm{FI})$, blood lactate concentration ([La]) measured 4 min postRAST and end-exercise ratings of perceived exertion (RPE)

Table 2 Running time and velocity, stride kinematics and spring-mass model during the RAST

\begin{tabular}{|c|c|c|c|c|c|c|c|}
\hline & Sprint 1 & Sprint 2 & Sprint 3 & Sprint 4 & Sprint 5 & Sprint 6 & ANOVA \\
\hline Time (s) & $4.89 \pm 0.14$ & $5.05 \pm 0.25$ & $5.31 \pm 0.22 * * *$ & $5.44 \pm 0.24 * * *$ & $5.62 \pm 0.33 * * *$ & $5.74 \pm 0.30^{* * *}$ & $P<0.001(+17.4 \%)$ \\
\hline$v\left(\mathrm{~m} \mathrm{~s}^{-1}\right)$ & $8.18 \pm 0.31$ & $7.84 \pm 0.46^{* *}$ & $7.48 \pm 0.40^{* * *}$ & $7.23 \pm 0.35^{* * *}$ & $6.88 \pm 0.44 * * *$ & $6.79 \pm 0.41^{* * *}$ & $P<0.001(-17.0 \%)$ \\
\hline \multicolumn{8}{|c|}{ Stride kinematics } \\
\hline$t_{\mathrm{c}}(\mathrm{ms})$ & $124 \pm 11$ & $123 \pm 7$ & $130 \pm 9$ & $132 \pm 13$ & $145 \pm 9 * * *$ & $148 \pm 9 * * *$ & $P<0.001(+20.0 \%)$ \\
\hline$t_{\mathrm{f}}(\mathrm{ms})$ & $110 \pm 15$ & $120 \pm 15$ & $120 \pm 19$ & $126 \pm 18$ & $123 \pm 13$ & $125 \pm 14$ & $P=0.176(+14.9 \%)$ \\
\hline$T_{\text {swing }}(\mathrm{ms})$ & $343 \pm 32$ & $358 \pm 32$ & $368 \pm 48$ & $385 \pm 48 *$ & $389 \pm 24 *$ & $398 \pm 35^{* *}$ & $P=0.002(+16.6 \%)$ \\
\hline$T_{\text {stride }}(\mathrm{ms})$ & $468 \pm 35$ & $485 \pm 30$ & $500 \pm 49$ & $516 \pm 45$ & $535 \pm 26$ & $545 \pm 41$ & $P<0.001(+17.0 \%)$ \\
\hline$S_{\mathrm{F}}(\mathrm{Hz})$ & $2.15 \pm 0.16$ & $2.07 \pm 0.13$ & $2.02 \pm 0.19$ & $1.95 \pm 0.18^{*}$ & $1.87 \pm 0.10^{* * *}$ & $1.84 \pm 0.14 * * *$ & $P<0.001(-13.9 \%)$ \\
\hline$S_{\mathrm{L}}(\mathrm{m})$ & $3.82 \pm 0.25$ & $3.81 \pm 0.37$ & $3.75 \pm 0.36$ & $3.74 \pm 0.41$ & $3.68 \pm 0.31$ & $3.70 \pm 0.39$ & $P=0.795(-2.9 \%)$ \\
\hline \multicolumn{8}{|c|}{ Spring-mass model } \\
\hline$F z_{\max }(\mathrm{N})$ & $2,361 \pm 180$ & $2,248 \pm 204$ & $2,320 \pm 215$ & $2,280 \pm 263$ & $2,425 \pm 218$ & $2,415 \pm 139$ & $P=0.145(+2.8 \%)$ \\
\hline$\Delta z(\mathrm{~cm})$ & $1.9 \pm 0.3$ & $1.8 \pm 0.2$ & $2.1 \pm 0.3$ & $2.1 \pm 0.4$ & $2.6 \pm 0.3 * * *$ & $2.7 \pm 0.3^{* * *}$ & $P<0.001(+45.7 \%)$ \\
\hline$\Delta L(\mathrm{~cm})$ & $17.5 \pm 2.5$ & $15.9 \pm 2.9$ & $16.4 \pm 2.1$ & $16.0 \pm 3.4$ & $17.8 \pm 2.9$ & $18.0 \pm 2.9$ & $P=0.268(+5.3 \%)$ \\
\hline$K_{\text {vert }}\left(\mathrm{kN} \mathrm{m}^{-1}\right)$ & $127.6 \pm 17.7$ & $123.1 \pm 14.0$ & $113.3 \pm 16.3$ & $108.5 \pm 16.4^{*}$ & $94.6 \pm 7.8 * * *$ & $91.4 \pm 10.4^{* * *}$ & $P<0.001(-27.2 \%)$ \\
\hline$K_{\mathrm{leg}}\left(\mathrm{kN} \mathrm{m}^{-1}\right)$ & $13.7 \pm 1.7$ & $14.5 \pm 3.0$ & $14.4 \pm 2.1$ & $14.7 \pm 2.9$ & $13.9 \pm 2.2$ & $13.8 \pm 2.7$ & $P=0.768(+1.5 \%)$ \\
\hline
\end{tabular}

Values are mean $\pm \mathrm{SD}$

$v$ running velocity, $t_{\mathrm{c}}$ contact time, $t_{\mathrm{f}}$ flight time, $T_{\text {swing }}$ swing time, $T_{\text {stride }}$ stride duration, $S_{\mathrm{F}}$ stride frequency, $S_{\mathrm{L}}$ stride length, $F z_{\text {max }}$ maximal vertical force, $\Delta \mathrm{z}$ centre of mass vertical displacement, $\Delta L$ leg compression, $K_{\text {vert }}$ vertical stiffness, $K_{\text {leg }}$ leg stiffness

* Significantly different from sprint $1, P<0.05$; ** $P<0.01$; *** $P<0.001$

ANOVA for repeated measures (sprint number) was used to compare changes in SMM, EMG and NIRS parameters with time. Multiple comparisons were made with the Tukey HSD post-hoc test when the Greenhouse-Geisser epsilon correction factor was $>0.50$, or with the Bonferroni post-hoc test when the epsilon was $<0.05$. Relationships between variables were examined using the Pearson product-moment correlation coefficient. Statistical analyses were undertaken by using the SigmaPlot version 11.0 Software (Systat Software, Inc., San Jose, CA, USA). Statistical significance was accepted at $P<0.05$.

\section{Results}

\section{RAST outcomes}

Averaged values for blood lactate concentration increased significantly $(P<0.001)$ from post- warming-up $\left(3.5 \pm 1.0 \mathrm{mmol} \mathrm{L}^{-1}\right)$ to $4 \mathrm{~min}$
post-RAST $\left(10.5 \pm 2.0 \mathrm{mmol} \mathrm{L}{ }^{-1}\right)$, while post-RAST RPE was $15.9 \pm 0.9$ a.u. (between 'hard' $=15$ and 'very hard' $=17$, on the Borg RPE scale). The Sdec was $9.5 \pm 2.4 \%$. RAST outcomes are shown in Table 1 . There was a significant increase in running times over the six sprints (from $4.89 \pm 0.14 \mathrm{~s}$ for the first sprint to $5.74 \pm 0.30 \mathrm{~s}$ for the last sprint; $+17.0 \% ; P<0.001$ ) (Table 2).

Sprinting mechanics

Stride kinematics and spring-mass characteristics values are displayed in Table 2: sprint time, $t_{\mathrm{c}}$, total stride duration and swing time increased from the first to the last repetition $(+17.4,+20.0,+17.0$ and $+16.6 \%$, respectively; all $P<0.05$ ), while $t_{\mathrm{f}}$ and $S_{\mathrm{L}}$ remained constant. $S_{\mathrm{F}}(-13.9 \% ; P<0.001)$ and $K_{\text {vert }}$ decreased $(-27.2 \%$; $P<0.001)$ across trials, whereas leg stiffness change was not significant $(+1.5 \% ; P>0.05)$. There was a strong 
correlation between $K_{\text {vert }}$ and $S_{\mathrm{F}}(r=0.91 ; P<0.01)$ and $S_{\mathrm{L}}(r=-0.76 ; P<0.05)$. Changes in $K_{\text {vert }}$ were primarily associated with changes in $\Delta z(r=-0.88 ; P<0.01)$, due to longer $t_{\mathrm{c}}(r=-0.88 ; P<0.01)$, and not to change in $F z_{\text {max }}(r=-0.11 ; P>0.05)$.

\section{NIRS}

The changes in TSI across sprint and recovery periods during the RAST and corresponding de- and re-oxygenation rates are displayed in Fig. 1. In reference to the initial sprint, VL muscle was further de-oxygenated by $18.6 \%$ at the end of the second sprint $(P<0.001)$, while no additional de-oxygenation was apparent thereafter $(-0.1 \%$ from sprint 2-6). In comparison to sprint 1 , faster de-oxygenation rates occurred during sprints 3-5 (Fig. 1). At the end of each recovery, TSI returned to end-recovery value of the previous sprint $(0.3 \%)$, with the exception of the first repetition $(-5.0 \%$ from baseline; $P<0.001)$. Mean (6 sprints) de-oxygenation and re-oxygenation rates were $1.52 \pm 0.87$ and $0.86 \pm 0.52 \% \mathrm{~s}^{-1}$, respectively.

Mean (6 sprints) re-oxygenation rates was negatively associated with cumulated sprint times $(r=-0.84$; $P<0.01$; Fig. 2$)$, mean power $(r=-0.79 ; P<0.05)$ and normalized mean power $(r=-0.84 ; P<0.05)$. Significant negative correlations were found between the mean (6 sprints) de-oxygenation rates and best sprint time $(r=-0.76 ; P<0.05)$, cumulated sprint times $(r=-0.74$; $P<0.05)$, mean power $(r=0.84 ; P<0.05)$ and mean normalized power $(r=0.84 ; P<0.05)$.

\section{EMG data}

Decreased RMS muscle activity occurred from the first to the last sprint for $\mathrm{RF}$ and $\mathrm{BF}(-18.7$ and $-18.1 \%$; $P<0.01$ and $P<0.001$, respectively), but not VL $(-1.2 \%$; $P>0.05$ ), muscles (Table 3).

Significant correlations were found between decline in RMS activities for RF and $\mathrm{BF}$ and increases in running times $(r=-0.82$ and -0.90 ; both $P<0.05$; Fig. 3 ). However, only BF muscle EMG activity correlated with power $(r=0.92 ; P<0.001)$ and normalized power $(r=0.81$; $P<0.01)$. Besides, fatigue index correlated with both $\mathrm{RF}$ and BF muscle activities $(r=-0.81$ and -0.82 , both $P<0.05$, respectively), as well as $S_{\mathrm{dec}}(r=0.88 ; P<0.01)$.

\section{Discussion}

Previous studies have mainly focused on biomechanical, neuromuscular and metabolic manifestations of fatigue during RSA tests in isolation often using non-elite sport populations. Therefore, by using non-invasive and portable

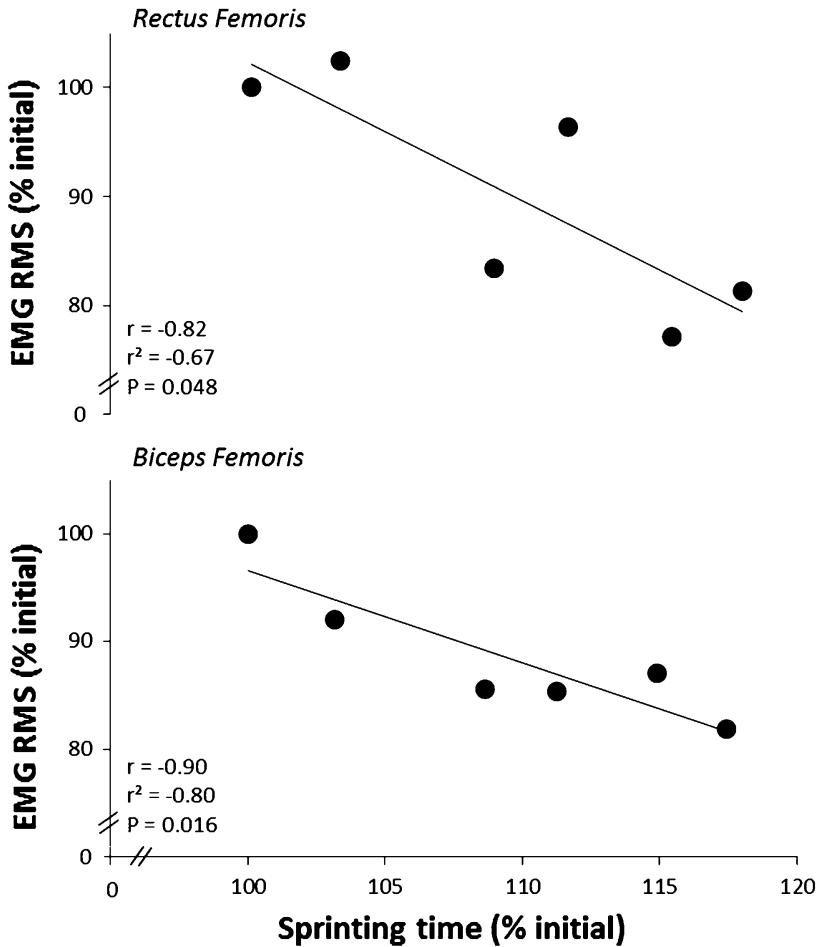

Fig. 2 Relationship between sprinting times during repetitions 1-6 (expressed as \% of the first sprint) and rectus femoris (top) and biceps femoris (bottom) muscles EMG amplitude (i.e. RMS; expressed as $\%$ of the first sprint) during the RAST ( $n=8$ for each data point)

devices and by adopting a highly ecological approach, the main originality of the present study was to simultaneously evaluate changes in leg-spring behaviour, knee extensor/ flexor muscle activation and VL muscle oxygenation trends during the so-called RAST. Slower times across the six 35-m sprints were accompanied by deteriorated lower-limb mechanical behaviour, characterized by progressive reductions in $K_{\text {vert }}$ values and less efficient stride characteristics (i.e. longer $t_{\mathrm{c}}$ and slower $S_{\mathrm{F}}$ ). Furthermore, players who maintained high activation levels in RF and BF muscles and displayed faster mean VL muscle re-oxygenation rates also had better RSA.

\section{RSA performance}

RAST outputs (peak power, mean power and fatigue index) and the pronounced end-exercise RPE score and blood lactate concentration $\left(>10 \mathrm{mmol} \mathrm{l}^{-1}\right.$ ) confirm that players reached a considerable degree of fatigue. The present data are consistent with those previously obtained with elite junior (Cipryan and Gajda 2011) or collegiate level football players (Keir et al. 2013), but better than U20 Brazilian footballers (Deminice et al. 2013), tackle football players from an NCAA Division I (Gwacham and Wagner 2012) or 
Table 3 Changes in root mean square (RMS) activity (\%) for vastus lateralis (VL), rectus femoris (RF) and biceps femoris (BF) muscles during the RAST

\begin{tabular}{llcccccc}
\hline & Sprint 1 & Sprint 2 & Sprint 3 & Sprint 4 & Sprint 5 & Sprint 6 & ANOVA \\
\hline VL & $100.0 \pm 0.0$ & $106.5 \pm 13.4$ & $92.1 \pm 19.9$ & $105.2 \pm 20.0$ & $90.8 \pm 19.7$ & $98.8 \pm 25.7$ & $P=0.064(-1.2 \%)$ \\
RF & $100.0 \pm 0.0$ & $102.4 \pm 19.5$ & $83 \pm 22.3$ & $96.4 \pm 35.2$ & $77.2 \pm 24.1$ & $81.3 \pm 28.1$ & $P=0.009(-18.7 \%)$ \\
BF & $100.0 \pm 0.0$ & $92.0 \pm 8.1$ & $85.6 \pm 11.5^{* * *}$ & $85.4 \pm 9.5^{* * *}$ & $87.1 \pm 11.0^{* *}$ & $81.9 \pm 12.1 * * *$ & $P<0.001(-18.1 \%)$ \\
\hline
\end{tabular}

Values are mean $\pm \mathrm{SD}$. For each muscle, RMS activity was normalized to the first sprint

** Significantly different from sprint $1, P<0.01$; *** $P<0.001$

Fig. 3 Relationship between mean (6 sprints) re-oxygenation rates and the cumulated sprint times during the RAST

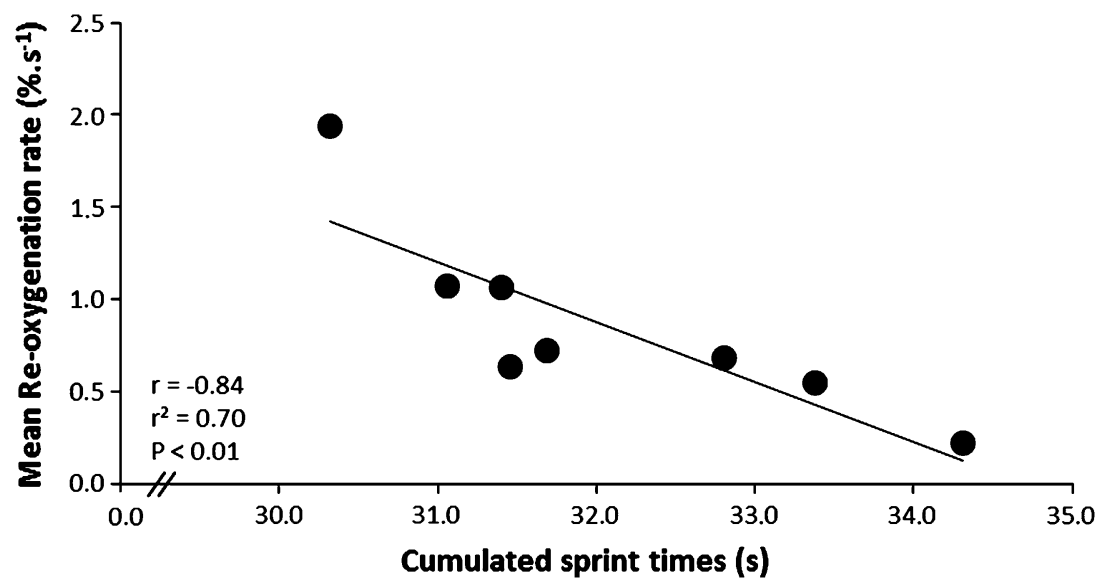

recreational middle-distance runners (Kaminagakura et al. 2012); i.e. all tested on an athletic track with the exception of Deminice et al. (2013) whose players wearing their football boots were tested on grass. In addition to participants' characteristics, it must be bear in mind that surface condition might have a significantly influence on anaerobic power production (and presumably on the magnitude of the accompanying neuro-mechanical and metabolic adjustments) during the RAST. Hence, $\sim 10 \%$ higher power values were registered in eight players completing the RAST test on a track (with sneakers) compared to grass (with football cleats) (Kalva-Filho et al. 2013). While testing football players in their usual training/competition environment enhances the ecological validity of our findings, the validity of the RAST to reflect fatigue during repeated high-intensity periods of a game has yet to be assessed.

Sprinting mechanics

We have recently investigated changes in sprinting mechanics occurring during a series of six 20-m sprints, separated by $20 \mathrm{~s}$ of passive recovery in a cohort of under- 19 football players tested in football-specific conditions; i.e. on natural grass with football boots (Girard et al. 2011c): progressive longer sprint times $(+6.7 \%)$ between the first and the sixth repetition were accompanied by a significant increase in $t_{\mathrm{c}}(+12.9 \%)$ and total stride durations $(+9.3 \%)$ together with reduction of $S_{\mathrm{F}}(-6.8 \%)$. With similar adjustments occurring during the RAST, this confirms that our players' ability to tolerate impact forces/stretch loads was also progressively impaired with sprint repetitions. Compared to Girard et al. (2011c), however, the more severe exerciseto-rest ratio when executing the RAST (i.e. 1:6 vs. 1:2 also corresponding to approximately twice longer sprints time and a two-fold decrease in recoveries duration) may have exacerbated the degree of fatigue, as confirmed by a larger $S_{\mathrm{dec}}(2.8 \pm 1.7 \%$ vs. $9.5 \pm 2.4 \%)$, and presumably the magnitude of accompanying mechanical adjustments. When sixteen team- and racquet-sports players performed $12 \times 40 \mathrm{~m}$ sprints interspersed with $30 \mathrm{~s}$ of passive recovery, the $S_{\text {dec }}(9.2 \pm 4.2 \%)$ and the magnitude of fatigueinduced changes in stride parameters at the 30-35-m distance interval were relatively similar to those reported here (Girard et al. 2011b). The inability to maintain constant performance (i.e. running velocity decreases across repetitions) during the RAST, however, makes it difficult to directly relate mechanical adjustments to fatigue resistance. Because modifications in running mechanics are highly influenced by running velocity changes, it can be argued that less efficient strides may not to be the cause but rather the effect of slower sprint times (Morin et al. 2007). Innovative approaches including a sprint-matching paradigm (Mendez-Villanueva et al. 2008) or measuring the recovery of performance and of its underpinning regulatory 
neuromuscular or metabolic factors (Mendez-Villanueva et al. 2012) are warranted to determine whether a disproportionate decreases in mechanical efficiency over running speed actually occur during RSA tests.

The SMM, which consists of a point mass bouncing on a linear spring, has contributed to improve our understanding of the effects of fatigue on the mechanics of sprinting (Brughelli and Cronin 2008). In basketball players tested on a non-motorized treadmill before and immediately after an official match, impaired RSA $(6 \times 4 \mathrm{~s}$ sprints with $21 \mathrm{~s}$ of passive recovery) was associated with a $4 \%$ reduction in $S_{\mathrm{F}}$ (Delextrat et al. 2013). More specific to our results, monotonic reductions in $K_{\text {vert }}$ across sprint repetitions have been documented during the course of various field-based RSA, while leg stiffness generally remains unchanged (Girard et al. 2011b, c; Morin et al. 2006). In line with these findings, we show that an increase in vertical motion of the centre of mass (while neither peak vertical forces nor leg compression present significant changes with fatigue), and hence a slower $S_{\mathrm{F}}$ (or longer $t_{\mathrm{c}}$ ) are connected to RAST performance. Nonetheless, there is no consensus in the literature as to whether $S_{\mathrm{L}}$ or $S_{\mathrm{F}}$ is the most critical factor affecting sprinting performance, as their relative importance is also likely to change during the different phases of a sprint (Nagahara et al. 2014). The possibility of a negative interaction between $S_{\mathrm{L}}$ and $S_{\mathrm{F}}$ has been suggested and arguably would depend on factors including leg length, height of take-off and vertical velocity at take-off (Hunter et al. 2004). In line with previous findings (Girard et al. 2011c), we observed that $S_{\mathrm{F}}$ decreased across repetitions, while $S_{\mathrm{L}}$ was well preserved. Thus, a better maintenance of $K_{\text {vert }}$ values under fatigue may improve RAST performance by minimizing the increase in $t_{\mathrm{c}}$ and hence the decrease in $S_{\mathrm{F}}$. While altering $S_{\mathrm{F}}$ can affect lower extremity posturing and joint kinetics, such compensation changes in ankle, knee and hip angles need to be further described using video tracking systems. Furthermore, both the total force production capability and-more importantly-the technical ability to maintain efficiently the horizontal force direction decrease when sprints are repeated with incomplete recoveries (Morin et al. 2011). Quantifying the effect of over-ground repeated-sprint fatigue on force-velocity relationships, as potentially derived from the ability to produce horizontal force during the first few meters of the acceleration phase of sprint running (Mendiguchia et al. 2014), may bring additional insights to the biomechanical features studied here.

\section{EMG}

Previous cycling RSA investigations revealed a consistent concurrent decline in power output and the amplitude of EMG signals across repetitions when $S_{\text {dec }}$ exceeds 8-10\%
(Girard et al. 2011a). Comparatively to mild fatigue condition $\left(S_{\mathrm{dec}} \sim 3 \%\right)$ where the amplitude of lower-limb EMG signals across running sprint repetitions was unaltered despite a significant deterioration in sprinting mechanics (Girard et al. 2011c), RAST completion resulted in substantial performance decrements $\left(S_{\mathrm{dec}}\right.$ approaching $\left.10 \%\right)$. This was accompanied by large reductions in RMS activity in RF and BF muscles, while no changes occurred in VL. While caution is needed when inferring motor control strategies from EMG (Farina et al. 2004), this could be viewed as a sign of altered RF and BF muscles coordination pattern and/or suboptimal net motor unit activity, due to progressive inhibition of motor units and/or the decrease in motor unit firing rate. Interestingly, these bi-articular hip-mobilizing muscles have already been shown to be the lower-limb muscles that present the earliest signs of change in their activity with fatigue (Hanon et al. 2005). The strong correlations found between changes in RF or BF RMS activity and sprint times confirm that performance during 'all-out' efforts with very short recoveries (10 s) largely rely on the maintenance of high motor unit activity.

Arguably, the inherent intense decelerations that accompany RAST completion could have increased the stress imposed on RF and BF muscles to a larger extent than on VL. It has recently been reported that a decline in the BF EMG activity was responsible for the reduction in eccentric knee flexor strength measured post-RSA $(6 \times 20 \mathrm{~m}$ over-ground sprints with a $10-\mathrm{m}$ acceleration distance and $15 \mathrm{~m}$ for deceleration; Timmins et al. 2014). In our study, the fatigue-induced lowering in BF muscle myoelectrical activity during the RAST is compatible with the view that the injury susceptibility of the hamstrings is high during the most intense phases of a football game (Dupont et al. 2010b). Reportedly, centrally mediated reductions in hamstring rate of torque development (during the first half) and maximal torque (latter stages of the half) have been reported during a 90-min simulated soccer match (Marshall et al. 2014). In fact, during the terminal swing phase of sprinting, hamstrings are undergoing an active lengthening (i.e. eccentric) contraction, and the combination of hip flexion and knee extension induces a large substantial local elongation stress on the myotendinous junctions and tendons [i.e. the most sensitive structures to strain (Guex and Millet 2013)]. During this phase, the hamstrings are therefore more vulnerable to injuries (Schache et al. 2012). While EMG activities have been calculated for the whole gait cycle in our study, it remains possible that fatigue-induced EMG reductions may have occurred at specific phases in one stride (i.e. pre-landing phase). This may particularly be true for the RF muscle during maximal sprinting, displaying a two-peak activity with the highest during the swing phase (as antagonist activity when BF had the lowest activity level) and a lower peak during 
the stance phase (when BF activity is maximal) (Jonhagen et al. 1996; Small et al. 2009). However, whether centrally programmed neuromuscular activity prior to the contact (i.e. high muscle pre-activation levels before and during the braking phase and short-latency stretch reflex responses; (Avela and Komi 1998)) is a decisive mechanism in the regulation of the stiffness behaviour of the lower limb during RSA similar to the RAST needs to be researched.

\section{NIRS}

We observed that during the RAST, a substantial and rapid decrease in VL muscle oxygenation levels during the initial sprint repetition as de-oxygenation in exercising muscles exceeds re-oxygenation (i.e. greater reliance on oxygen extraction). It is well described that there is a time delay of several seconds (not quantified here) in muscle re-oxygenation after the completion of maximal-intensity exercise (Ihsan et al. 2013), which may explain why after only $10 \mathrm{~s}$ of recovery muscle re-oxygenation is partial. Following a similar trend than sprinting performance, muscle de-oxygenation rates became progressively lower during successive sprint repetitions in reference to the first sprint, whereas muscle re-oxygenation rates displayed an opposite pattern. In the absence of direct measurement of local oxygen uptake in the active musculature, whether it is more the rate of muscle blood flow or the $\mathrm{O}_{2}$ delivery/utilization ratio that primarily determines the sprint-induced decline in deoxygenation capacity across repetition cannot be ascertain.

To our knowledge, this study represents the first major attempt to carefully measure muscle re-oxygenation rates during repeated maximal running sprints in a highly ecological test setting and to report relationships with players' RSA. An important finding of this study was that players with a better repeated-sprint performance (lower cumulated times) had faster muscle de- (during sprints) and reoxygenation (during recovery) rates during the RAST. Our results support the latest methodological recommendation to use rates-instead of maximal values - of re-oxygenation (Ihsan et al. 2013) and suggest that the level of muscle oxygenation during the recovery periods between sprints (i.e. muscle re-oxygenation capacity) has a large influence on the ability to maintain performance. In contrast, when ten male team-sport athletes performed 6 repeated maximal 4-s sprints interspersed with $21 \mathrm{~s}$ of passive recovery on a non-motorized treadmill, it was suggested that "for very short run-based repeated sprints, the level of muscle oxygenation reached might not be as important to preserve RSA" (Buchheit et al. 2009). Other studies also failed to observe significant relationships between muscle re-oxygenation rates and running $(2 \times 15 \mathrm{~m}$ shuttle sprints- $15 \mathrm{~s}$ of passive recovery; (Buchheit and Ufland 2011) or cycling $(6 \times 30 \mathrm{~s}$ 'all-out' sprints-2 min of passive recovery;
Buchheit et al. 2012) RSA tests. These discrepant findings may partly relate to the wide variety of exercise protocols (emphasizing the importance of the work-to-rest ratios) or modes (cycling vs. running), oxygenation-derived variables (de-oxyhemoglobin vs. TSI) or analysis methods implemented (linear modelling, minimum and maximum or mean values during work/rest intervals). Bearing in mind that those individuals with a larger proportion of glycolytic fibres also display accelerated muscle re-oxygenations after sprint exercise compared to their aerobically fit counterparts (Nagasawa 2013), position-specific physical demands (Ingebrigtsen et al. 2014) and heterogeneous anthropometric and/or neuromuscular (Rey et al. 2012) profiles or training status generally characterizing football players may also account for the different relationships between RSA and muscle oxygenation trends. In the same group of players from the Qatar national football team, we have recently observed that power-related qualities and RAST performance are positively associated with a high muscular profile and a low adiposity (Brocherie et al. 2014b). This may have strengthened the link between RSA and re-oxygenation rates in this cohort of professional players.

\section{Limitations}

Several limitations need to be highlighted. First, our NIRS measurements were taken over the most proximal parts of the VL muscle $(10 \mathrm{~cm}$ above the proximal border of the patella), when EMG recordings are simultaneously under investigation. However, substantial heterogeneity exists within the quadriceps with respect to the dynamic response of muscle de-oxygenation following the onset of moderate and heavy cycling exercise (Koga et al. 2007), potentially supporting the use of multichannel NIRS to better represent spatial and temporal muscle de-/re-oxygenation profiles during RSA tests. Moreover, the lack of change in VL EMG activation, while substantial reductions occurred in both RF and $\mathrm{BF}$ muscles gives support to investigate oxygenation trends in these later lower-limb muscles during RSA tests since perturbations in their re-oxygenation capacities could negatively impact mechanical behaviour and ultimately performance. Second, there are many complex methodological (electrode placement and shift, signal crosstalk from other muscles) and physiological (signal non-stationarity, subcutaneous tissue, blood flow, fibre diameter, muscle biochemistry) factors, unrelated to the level of neural activation, that can influence the surface EMG signal. It is therefore difficult to definitively conclude that neural input reaching the neuromuscular junction of RF and BF muscles effectively decreased across repetitions during the RAST. A valuable normalization procedure would be to report the EMG signal as a ratio of the actual size of a maximal compound muscle action potential (or M-wave), which is 
commonly been used as an indirect index of muscle excitability. While this methodological precaution is now used routinely in most 'fatigue' studies involving isolated contractions, the methodological problems of quantifying EMG-based indices of central motor output adjustments during dynamic, whole-body exercises need to be considered in future investigations. Third, when investigating neuro-mechanical adjustments during the $35 \mathrm{~m}$ runs we were specifically focusing our analysis on the $25-30-\mathrm{m}$ distance interval so as to picture the three steps where players produced the largest mechanical power output. The validity of this approach is supported by Racinais et al. (2007) who reported that VL RMS activity is significantly less during the second and the third pedal revolutions during a RSA test $(10 \times 6$-s cycling sprints with $30 \mathrm{~s}$ rest $)$, while not change could be detected when the whole 6-s sprint duration was considered. As such, our results must remain specific to the 25-30-m distance interval of the $35 \mathrm{~m}$ sprint and should not be extrapolated to other phases of the sprint (i.e. early acceleration).

\section{Practical applications}

While the clinical relevance of improving RSA in football is a matter of debate (Bishop et al. 2011; Buchheit 2012; Carling 2013), football players experience temporary fatigue during a game (e.g. lower amount of accelerations, sprinting after repeated and/or intense actions) (Mohr et al. 2005) which may negatively determine the outcome of crucial phases of the game. By strengthening the nature of the relationships between RAST outcomes and the magnitude of accompanying neuro-mechanical and metabolic adjustments, our results lend support to several practical implications. First, since the capacity of the neuromuscular system to fully activate the lower limb (at least the RF muscle), leading to more 'powerful' strides, is closely related to the ability to produce and maintain maximal sprinting speeds implementing resistance training regimens (e.g. plyometrics, eccentric strength, rapid muscle force production executed with systemic hypoxia or with blood flow restriction to the working muscles) is a promising approach. In this context, the prescription of resistance multi-joint exercises under hypoxic conditions is expected to result in larger strength gains than equivalent training in normoxia, through an up-regulated neuromuscular control (i.e. greater muscle activation and facilitated recruitment of type II muscle fibres) and augmented muscular changes (i.e. oxygen delivery, intramuscular signalling pathways) (Scott et al. 2014). Training under these circumstances would potentially improve musculoskeletal stiffness regulation (i.e. minimal increases in contact times and hence decreases in stride frequencies) when players experience fatigue and thereby improve RAST outcomes, while evidence for such assumption is still lacking. Second, improving re-oxygenation capacity in the lower-limb muscles during post-sprint recovery periods would likely participate to the better maintenance of repeated-sprint performance. In this vein, accelerated post-sprint re-oxygenation rates and/or blood perfusion have been reported over the duration of a competitive season in football (Hoffman et al. 2005), following a 8-weeks 'endurance-oriented' training programme (Buchheit and Ufland 2011) and after 4-weeks hypoxic training (Faiss et al. 2013; Kime et al. 2003). More specific to team sports, improvements in VL muscle re-oxygenation rates were possible in elite UK premiership academy level rugby players following supra-maximal cycling for 8 weeks (ten 10-s cycle sprints interspersed with $40 \mathrm{~s}$ recovery; Jones and Cooper 2014). In youth highly trained football players, the addition of ten repeated-sprint training sessions performed in hypoxia vs. normoxia to their regular football practice over a 5-week in-season period was more efficient at enhancing repeated-agility ability (including direction changes) (Brocherie et al. 2014a).

\section{Conclusion}

In professional football players, we showed that repeated sprinting $(6 \times 35 \mathrm{~m}-10 \mathrm{~s}$ recovery $)$ with players wearing their football boots on grass induces profound impairments in leg-spring behaviour and stride mechanics. Our results also strengthen the link between RSA and the alteration magnitude of both lower limb neuromuscular activation and muscle de- and re-oxygenation rates.

Acknowledgments The authors are grateful to the Qatar Football Association for their support and the national team players for their enthusiastic participation.

\section{References}

Avela J, Komi PV (1998) Interaction between muscle stiffness and stretch reflex sensitivity after long-term stretch-shortening cycle exercise. Muscle Nerve 21:1224-1227

Balciunas M, Stonkus S, Abrantes C, Sampaio J (2006) Long term effects of different training modalities on power, speed, skill and anaerobic capacity in young male basketball players. J Sports Sci Med 5:163-170

Beck WR, Zagatto AM, Gobatto CA (2014) Repeated sprint ability tests and intensity-time curvature constant to predict short-distance running performances. Sport Sci Health 10:105-110

Bishop D, Girard O, Mendez-Villanueva A (2011) Repeated-sprint ability - part II: recommendations for training. Sports Med 41:741-756

Borg G (1970) Perceived exertion as an indicator of somatic stress. Scand J Rehabil Med 2:92-98

Brocherie F, Girard O, Faiss R, Millet GP (2014a) High-intensity intermittent training in hypoxia: a double-blinded, placebo-controlled field study in youth football players J Strength Cond Res [Epub ahead of print] 
Brocherie F, Girard O, Forchino F, Al Haddad H, Dos Santos G, Millet GP (2014b) Relationships between anthropometric measures and athletic performance, with special reference to repeatedsprint ability, in the Qatar national soccer team. J Sports Sci 32:1243-1254

Brughelli M, Cronin J (2008) Influence of running velocity on vertical, leg and joint stiffness: modelling and recommendations for future research. Sports Med 38:647-657

Buchheit M (2012) Fatigue during repeated sprints: precision needed. Sports Med 42:165-167

Buchheit M, Ufland P (2011) Effect of endurance training on performance and muscle reoxygenation rate during repeated-sprint running. Eur J Appl Physiol 111:293-301

Buchheit M, Cormie P, Abbiss CR, Ahmaidi S, Nosaka KK, Laursen PB (2009) Muscle deoxygenation during repeated sprint running: effect of active vs. passive recovery. Int J Sports Med 30:418-425

Buchheit M, Bishop D, Haydar B, Nakamura FY, Ahmaidi S (2010) Physiological responses to shuttle repeated-sprint running. Int $\mathbf{J}$ Sports Med 31:402-409

Buchheit M, Abbiss CR, Peiffer JJ, Laursen PB (2012) Performance and physiological responses during a sprint interval training session: relationships with muscle oxygenation and pulmonary oxygen uptake kinetics. Eur J Appl Physiol 112:767-779

Carling C (2013) Interpreting physical performance in professional soccer match-play: should we be more pragmatic in our approach? Sports Med 43:655-663

Cipryan L, Gajda V (2011) The influence of aerobic power on repeated anaerobic exercise in junior soccer players. J Hum Kinet 28:63-71

Coleman DR, Cannavan D, Horne S, Blazevich AJ (2012) Leg stiffness in human running: comparison of estimates derived from previously published models to direct kinematic-kinetic measures. J Biomech 45:1987-1991

Delextrat A, Baliqi F, Clarke N (2013) Repeated sprint ability and stride kinematics are altered following an official match in national-level basketball players. J Sports Med Phys Fit 53:112-118

Deminice R, Rosa FT, Franco GS, Jordao AA, de Freitas EC (2013) Effects of creatine supplementation on oxidative stress and inflammatory markers after repeated-sprint exercise in humans. Nutrition 29:1127-1132

Dupont G, McCall A, Prieur F, Millet GP, Berthoin S (2010a) Faster oxygen uptake kinetics during recovery is related to better repeated sprinting ability. Eur J Appl Physiol 110:627-634

Dupont G, Nedelec M, McCall A, McCormack D, Berthoin S, Wisloff $\mathrm{U}$ (2010b) Effect of 2 soccer matches in a week on physical performance and injury rate. Am J Sports Med 38:1752-1758

Faiss R, Leger B, Vesin JM, Fournier PE, Eggel Y, Deriaz O, Millet GP (2013) Significant molecular and systemic adaptations after repeated sprint training in hypoxia. PLoS ONE 8:e56522

Farina D, Merletti R, Enoka RM (2004) The extraction of neural strategies from the surface EMG. J Appl Physiol 96:1486-1495

Faude O, Koch T, Meyer T (2012) Straight sprinting is the most frequent action in goal situations in professional football. J Sports Sci 30:625-631

Ferrari M, Muthalib M, Quaresima V (2011) The use of near-infrared spectroscopy in understanding skeletal muscle physiology: recent developments. Philos Trans A 369:4577-4590

Girard O, Mendez-Villanueva A, Bishop D (2011a) Repeated-sprint ability-part I: factors contributing to fatigue. Sports Med 41:673-694

Girard O, Micallef JP, Millet GP (2011b) Changes in spring-mass model characteristics during repeated running sprints. Eur J Appl Physiol 111:125-134

Girard O, Racinais S, Kelly L, Millet GP, Brocherie F (2011c) Repeated sprinting on natural grass impairs vertical stiffness but does not alter plantar loading in soccer players. Eur J Appl Physiol 111:2547-2555

Glaister M, Howatson G, Pattison JR, McInnes G (2008) The reliability and validity of fatigue measures during multiple-sprint work: an issue revisited. J Strength Cond Res 22:1597-1601

Guex K, Millet GP (2013) Conceptual framework for strengthening exercises to prevent hamstring strains. Sports Med 43:1207-1215

Gwacham N, Wagner DR (2012) Acute effects of a caffeine-taurine energy drink on repeated sprint performance of American college football players. Int J Sport Nutr Exerc Metab 22:109-116

Hanon C, Thepaut-Mathieu C, Vandewalle H (2005) Determination of muscular fatigue in elite runners. Eur J Appl Physiol 94:118-125

Hoffman JR, Im J, Kang J, Ratamess NA, Nioka S, Rundell KW, Kime R, Cooper J, Chance B (2005) The effect of a competitive collegiate football season on power performance and muscle oxygen recovery kinetics. J Strength Cond Res 19:509-513

Hunter JP, Marshall RN, McNair PJ (2004) Interaction of step length and step rate during sprint running. Med Sci Sports Exerc 36:261-271

Ihsan M, Abbiss CR, Lipski M, Buchheit M, Watson G (2013) Muscle oxygenation and blood volume reliability during continuous and intermittent running. Int J Sports Med 34:637-645

Ingebrigtsen J, Dalen T, Hjelde GH, Drust B, Wisloff U (2014) Acceleration and sprint profiles of a professional elite football team in match play. Eur J Sport Sci 1-10 [Epub ahead of print]

Jones B, Cooper CE (2014) Use of NIRS to assess effect of training on peripheral muscle oxygenation changes in elite rugby players performing repeated supramaximal cycling tests. Adv Exp Med Biol 812:333-339

Jonhagen S, Ericson MO, Nemeth G, Eriksson E (1996) Amplitude and timing of electromyographic activity during sprinting. Scand J Med Sci Sports 6:15-21

Kalva-Filho CA, Loures JP, Franco GS, Kaminagakura EI, Zagatto AM, Papoti M (2013) Comparison of the anaeorobic power measured by the RAST test at different footwear and surfaces conditions. Rev Bras Med Esporte 19:139-142

Kaminagakura EI, Zagatto AM, Redkva PE, Gomes EB, Loures JP, Kalva-Filho CA, Papoti M (2012) Can the running-based anaerobic sprint test be used to predict anaerobic capacity? J Exerc Physiol 15:90-99

Keir DA, Theriault F, Serresse O (2013) Evaluation of the runningbased anaerobic sprint test as a measure of repeated sprint ability in collegiate-level soccer players. J Strength Cond Res 27:1671-1678

Kelly LA, Racinais S, Tanner CM, Grantham J, Chalabi H (2010) Augmented low dye taping changes muscle activation patterns and plantar pressure during treadmill running. J Orthop Sports Phys Ther 40:648-655

Kime R, Karlsen T, Nioka S, Lech G, Madsen O, Saeterdal R, Im J, Chance B, Stray-Gundersen J (2003) Discrepancy between cardiorespiratory system and skeletal muscle in elite cyclists after hypoxic training. Dyn Med 2:4

Koga S, Poole DC, Ferreira LF, Whipp BJ, Kondo N, Saitoh T, Ohmae E, Barstow TJ (2007) Spatial heterogeneity of quadriceps muscle deoxygenation kinetics during cycle exercise (1985). J Appl Physiol 103:2049-2056

Marshall PW, Lovell R, Jeppesen GK, Andersen K, Siegler JC (2014) Hamstring muscle fatigue and central motor output during a simulated soccer match. PLoS One 9:e102753

McCully KK, Iotti S, Kendrick K, Wang Z, Posner JD, Leigh J Jr, Chance B (1994) Simultaneous in vivo measurements of $\mathrm{HbO}_{2}$ saturation and $\mathrm{PCr}$ kinetics after exercise in normal humans. J Appl Physiol 77:5-10

Mendez-Villanueva A, Hamer P, Bishop D (2008) Fatigue in repeatedsprint exercise is related to muscle power factors and reduced neuromuscular activity. Eur J Appl Physiol 103:411-419 
Mendez-Villanueva A, Edge J, Suriano R, Hamer P, Bishop D (2012) The recovery of repeated-sprint exercise is associated with $\mathrm{PCr}$ resynthesis, while muscle $\mathrm{pH}$ and EMG amplitude remain depressed. PLoS One 7:e51977

Mendiguchia J, Samozino P, Martinez-Ruiz E, Brughelli M, Schmikli S, Morin JB, Mendez-Villanueva A (2014) Progression of mechanical properties during on-field sprint running after returning to sports from a hamstring muscle injury in soccer players. Int J Sports Med 114(11):2281-2288

Mohr M, Krustrup P, Bangsbo J (2005) Fatigue in soccer: a brief review. J Sports Sci 23:593-599

Morin JB, Seve P (2011) Sprint running performance: comparison between treadmill and field conditions. Eur J Appl Physiol 111:1695-1703

Morin JB, Dalleau G, Kyrolainen H, Jeannin T, Belli A (2005) A simple method for measuring stiffness during running. J Appl Biomech 21:167-180

Morin JB, Jeannin T, Chevallier B, Belli A (2006) Spring-mass model characteristics during sprint running: correlation with performance and fatigue-induced changes. Int $\mathrm{J}$ Sports Med 27:158-165

Morin JB, Samozino P, Zameziati K, Belli A (2007) Effects of altered stride frequency and contact time on leg-spring behavior in human running. J Biomech 40:3341-3348

Morin JB, Samozino P, Edouard P, Tomazin K (2011) Effect of fatigue on force production and force application technique during repeated sprints. J Biomech 44:2719-2723

Nagahara R, Naito H, Morin JB, Zushi K (2014) Association of acceleration with spatiotemporal variables in maximal sprinting. Int $\mathrm{J}$ Sports Med 35:755-761

Nagasawa T (2013) Slower recovery rate of muscle oxygenation after sprint exercise in long-distance runners compared with that in sprinters and healthy controls. J Strength Cond Res 27:3360-3366
Racinais S, Bishop D, Denis R, Lattier G, Mendez-Villaneuva A, Perrey S (2007) Muscle deoxygenation and neural drive to the muscle during repeated sprint cycling. Med Sci Sports Exerc 39:268-274

Rey E, Lago-Penas C, Lago-Ballesteros J (2012) Tensiomyography of selected lower-limb muscles in professional soccer players. J Electromyogr Kinesiol 22:866-872

Schache AG, Dorn TW, Blanch PD, Brown NA, Pandy MG (2012) Mechanics of the human hamstring muscles during sprinting. Med Sci Sports Exerc 44:647-658

Scott BR, Slattery KM, Sculley DV, Dascombe BJ (2014) Hypoxia and resistance exercise: a comparison of localized and systemic methods. Sports Med 44(8):1037-1054

Small K, McNaughton LR, Greig M, Lohkamp M, Lovell R (2009) Soccer fatigue, sprinting and hamstring injury risk. Int J Sports Med 30:573-578

Spencer M, Bishop D, Dawson B, Goodman C (2005) Physiological and metabolic responses of repeated-sprint activities: specific to field-based team sports. Sports Med 35:1025-1044

Timmins RG, Opar DA, Williams MD, Schache AG, Dear NM, Shield AJ (2014) Reduced biceps femoris myoelectrical activity influences eccentric knee flexor weakness after repeat sprint running. Scand J Med Sci Sports. doi:10.1111/sms.12171

Winter DA (1990) Biomechanics and motor control of human movement, 2nd edn. Wiley Inter Science, New York

Wolf M, Ferrari M, Quaresima V (2007) Progress of near-infrared spectroscopy and topography for brain and muscle clinical applications. J Biomed Opt 12:062104

Zagatto AM, Beck WR, Gobatto CA (2009) Validity of the running anaerobic sprint test for assessing anaerobic power and predicting short-distance performances. J Strength Cond Res 23:1820-1827 